\title{
BMJ Open Interventions for the well-being of healthcare workers during a pandemic or other crisis: scoping review
}

Patrick Cairns (D) , ${ }^{1}$ Gill Aitken, ${ }^{2}$ Lindsey Margaret Pope (D) , ${ }^{3}$ Joanne E Cecil (D) , ${ }^{1}$ Kathryn B Cunningham, ${ }^{1}$ Julie Ferguson, ${ }^{4}$ Katie Gibson Smith, ${ }^{5}$ Lisi Gordon, ${ }^{6,7}$ Peter Johnston (D) , ${ }^{5,8}$ Anita Laidlaw (D) , ${ }^{1}$ Gillian Marion Scanlan, ${ }^{6}$ Tricia R Tooman (D) , ${ }^{1}$ Judy Wakeling, ${ }^{9}$ Kim Walker (1) ${ }^{5}$

To cite: Cairns $P$, Aitken $\mathrm{G}$, Pope LM, et al. Interventions for the well-being of healthcare workers during a pandemic or other crisis: scoping review. BMJ Open 2021;11:e047498. doi:10.1136/ bmjopen-2020-047498

- Prepublication history and additional supplemental material for this paper are available online. To view these files, please visit the journal online. (http://dx.doi.org/10.1136/ bmjopen-2020-047498).

Received 30 November 2020 Accepted 03 August 2021

Check for updates

(C) Author(s) (or their employer(s)) 2021. Re-use permitted under CC BY-NC. No commercial re-use. See rights and permissions. Published by BMJ.

For numbered affiliations see end of article.

Correspondence to

Patrick Cairns;

pc74@st-andrews.ac.uk

\section{ABSTRACT}

Objectives The aim of this scoping review was to identify pre-existing interventions to support the well-being of healthcare workers during a pandemic or other crisis and to assess the quality of these interventions.

Design Arksey and 0'Malley's five-stage scoping review framework was used to identify the types of evidence available in the field of well-being interventions for healthcare workers during a pandemic. PubMed, PsycINF0, Embase, Scopus, Web of Science, CINAHL and ERIC databases were searched to find interventions for the well-being of doctors during pandemics. Owing to a lack of results, this search was expanded to all healthcare workers and to include any crisis. Databases were searched in June 2020 and again in October 2020. Inclusion/exclusion criteria Articles were included that studied healthcare workers, reported an intervention design and were specifically designed for use during a pandemic or other crisis. Well-being was defined broadly and could include psychological, physical, social or educational interventions.

Results Searching produced 10529 total academic references of which 2062 were duplicates. This left 8467 references. Of these, 16 met our inclusion criteria and were included in data extraction. During data extraction, three more papers were excluded. This left 13 papers to summarise and report. Of these 13 papers, 6 were prospective studies and 7 were purely descriptive. None of the interventions were theoretically informed in their development and the quality of the evidence was generally deemed poor.

Conclusions There are no high-quality, theory-based interventions for the well-being of healthcare workers during a pandemic or other crisis. Given that previous pandemics have been shown to have a negative effect on healthcare workers well-being, it is imperative this shortcoming is addressed. This scoping review highlights the need for high-quality, theory-based and evidencebased interventions for the well-being of healthcare workers during a pandemic.

\section{INTRODUCTION}

As the world grapples with the SARS-CoV-2 pandemic (hereafter COVID-19), healthcare systems and workers are placed under
Strengths and limitations of this study

- This scoping review used a transparent methodological approach supported by the application of an established methodological framework.

- This review only included articles published in English, and well-being interventions for healthcare workers may exist in articles written in different languages.

- The searching was comprehensive, including seven academic bibliographic databases, three grey literature databases and one internet search engine.

- Our search terms were designed to be exhaustive but other search terms may exist that could produce further findings.

- A social media call was undertaken in an effort to further enhance the scope of the search.

increasing strain. ${ }^{1}{ }^{2}$ Teams are required to care for growing numbers of patients infected with a new and poorly understood disease. This work is often undertaken in challenging conditions and healthcare workers (HCWs) may consequently experience traumatic events. ${ }^{2} 2$ During previous disease outbreaks, such as severe acute respiratory syndrome (SARS), HCWs experienced feelings of extreme vulnerability and uncertainty producing somatic and cognitive symptoms of anxiety. ${ }^{3}{ }^{4}$ Following the control of the SARS outbreak, depression and avoidance were evident among HCWs, with the prevalence of psychiatric morbidity estimated at approximately $75 \% .^{34}$

During the COVID-19 pandemic, HCWs internationally have experienced increased depressive symptoms, anxiety, psychological distress and poor sleep quality. ${ }^{34}$ There is no determined definition for well-being, which can focus on multiple, different facets depending on the context and discipline. However, having identified that HCWs 
are negatively affected both physically (exposure to COVID-19, sleep loss and exhaustion) and psychologically (anxiety, depression and distress) by the COVID-19 pandemic, it is important to identify measures to support these aspects of HCWs' well-being during this time. The occurrence of previous disease outbreaks such as SARS, Ebola and H1N1 led to expectations that previous interventions designed to support HCWs during a pandemic may exist within the academic literature.

However, it was expected that different healthcare professions would experience pandemics differently, have different needs, differing decision making, varying roles and responsibilities and therefore separate literature would exist for each group. Thus, the purpose of this scoping literature review was to identify the types of interventions previously utilised to support the well-being of doctors during pandemics existing within the literature.

Synthesising the literature in this way would provide a singular evidence base from which interventions can be judged as effective or ineffective in supporting the well-being of doctors. This would ensure that the most effective interventions are used and that they are targeted appropriately. For the interventions within the literature to be used in practice, they should be of high quality and theory-based. ${ }^{5}$

Therefore, our research question was 'What interventions currently exist that support the well-being of doctors during pandemics?'.

\section{METHODS}

\section{Study design}

A scoping review methodology was chosen to provide an overview of the evidence rather than answering a specific question to inform policy or practice as is done in a systematic review. ${ }^{6}$ Arksey and $\mathrm{O}^{\prime}$ Malley's ${ }^{7}$ methodological framework was followed for this scoping review by identifying the research question, identifying relevant studies, selecting these studies, charting the data and collating, summarising and reporting the results. The scoping review also carried out according to the 'Preferred Reporting Items for Systematic Reviews and Meta-Analyses for scoping reviews' guidelines. ${ }^{8}$

\section{Research question}

Our initial research question was 'What interventions currently exist that support the well-being of doctors during pandemics?'.

As the search progressed, it became evident that the existing literature relating to interventions for doctors during pandemics either did not exist or was very small. It was therefore decided to extend the study population to HCWs and look beyond pandemics to other crises.

Therefore, our final research question was 'What interventions currently exist that support the well-being of healthcare workers during pandemics or other crises?'.

\section{Search strategy}

The following databases were searched: PubMed, PsycINFO, Embase, Scopus, Web of Science, CINAHL

\section{Box 1 Search terms used for each data base.}

((Epidemic* OR Pandemic* OR Human Influenza OR Disease Outbreaks OR Smallpox OR Dengue Virus OR Middle East Respiratory Syndrome Coronavirus OR HIV Infections OR Coronavirus Infections OR SARSCoV-2.mp OR COVID-19.mp OR Porcine Respiratory Coronavirus OR Ebola Haemorrhagic Fever OR ebola.mp OR H1N1 OR "Swine flu" OR Viral Pneumonia OR disaster* OR catastrophe OR crisis OR crises) AND

("health personnel" OR physician OR doctor*) AND

(Psychological Stress OR Physiological Stress OR Occupational Stress OR Psychological Burnout OR Professional Burnout OR Depression OR Health Promotion OR Self Care OR Mental Health OR Workload OR Suicide OR Fear OR Emotions OR Mental Health OR Post-Traumatic Stress Disorders OR Wounds and Injuries OR Dehydration OR Hunger OR Sleep))

and ERIC. This list includes all relevant databases that were selected to be as comprehensive as possible in our searching. No limits on date, country, subject or research type were placed on the database search, however only articles in English were considered during study selection.

The grey literature was explored to evaluate evidence from books, conference reports, academic preprints and government reports. The following grey literature databases were searched: OpenGrey, WorldCat, Medrxiv and Advanced Google Search. Results from the Advanced Google Search were limited to .org websites and .PDF files in a bid to find reports from well-being or healthcare organisations. An appeal on social media (Twitter) was also made to contact relevant researchers and experts to request any literature on pre-existing interventions that had not been published in the academic literature.

Search terms were selected to maximise the possibility of finding relevant articles and were developed with the support of a librarian. Box 1 depicts the search terms used for each data base. For online precise search strategy see appendices.

The search of the seven academic databases and grey literature sources was conducted in June 2020 with a follow-up search in October 2020 to identify any additional interventions published since the original search.

\section{Charting the data}

All citations were imported into bibliographic manager EndNote X9 (Clarivate Analytics, Philadelphia, PA, USA), and duplicate citations were removed. References were reviewed using their titles and abstracts, and full papers were reviewed when the relevance of the article was unclear. The included papers were then read in detail and a data capture form was developed and used to chart information including participants, type of intervention, theory used, quality assessment, outcome measures and results (see online supplemental appendix A for full data capture form). The data capture form was pretested prior to usage, and the data from a subsection of the included papers were extracted by three independent researchers. 


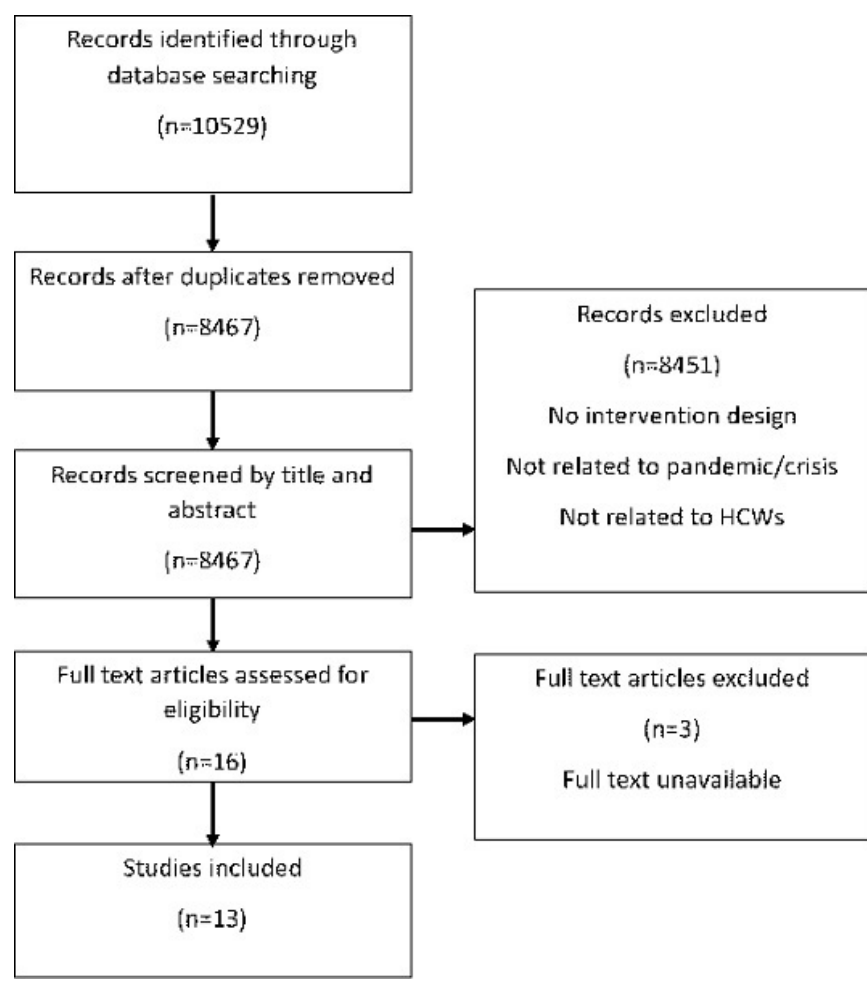

Figure 1 Preferred Reporting Items for Systematic Reviews and Meta-Analyses flowchart of academic study selection process.

Where papers adhered to established research types (eg, randomised controlled trials (RCTs) and prospective trials), quality assessment tools were used. Specifically, the National Institutes of Health Quality Assessment Tool for Before-After (Pre-Post) Studies With No Control Group was used for studies measuring effectiveness. ${ }^{9}$ Resources obtained from Twitter were collated separately and assessed the following: name, web link, country, intended audience, theory, issues address and status (see online supplemental appendix B).

\section{Study selection}

Studies were excluded if they did not include HCWs, involve an intervention, or relate to pandemics or other crises. Searching the seven academic databases produced a total of 10529 references. Of these, 2062 were duplicates. This left 8467 references. Of these, 16 papers fitting the inclusion criteria were chosen for data extraction. During data extraction, three more papers were excluded as the full text article was not available. This left 13 papers to synthesise, summarise and report (figure 1).

The grey literature contained 2325 total references, none of which were chosen for data extraction since they did not meet inclusion criteria.

\section{RESULTS}

\section{Types of publications found}

Of the 13 publications discovered from academic databases, none were RCTs. The 13 publications included 6 prospective studies ${ }^{10-15}$ and 7 descriptive studies ${ }^{16-22}$ resembling protocols. The general quality of the studies therefore ranged from poor to fair according to the hierarchy of scientific evidence. ${ }^{23}$ The studies were published between 2006 and 2020 and were conducted in Canada, China, France, Japan, Philippines, Taiwan, UK and USA (see tables 1 and 2).

\section{Prospective studies}

Details of the six prospective studies ${ }^{10-15}$ are provided in box 1. All interventions targeted psychological wellbeing. ${ }^{10-15}$ Four interventions targeted the psychological well-being of HCWs in general, ${ }^{10111314}$ one focused on the well-being of nurses ${ }^{11}$ and one focused on the well-being of disaster mental health workers. ${ }^{15}$ The contents of these interventions included education provision, ${ }^{10}{ }^{11}$ environmental restructuring, ${ }^{12}$ meditation ${ }^{13}{ }^{15}$ or a mixture of education and meditation. ${ }^{14}$ One study assessed acceptability of the intervention ${ }^{11}$ and five measured intervention effectiveness. ${ }^{10}{ }^{12-15}$ Outcome measures included Likert scales of the authors own creation ${ }^{1011} 13$ or a broad range of anxiety, depression, mood and sleep scales. ${ }^{12} 1315$

All interventions reported positive outcomes, ${ }^{10-15}$ including participants feeling better able to cope and more prepared to deal with a pandemic, ${ }^{10}$ improved anxiety and reduced depression, ${ }^{12-15}$ reduced PTSD $^{14}$ and a perception of usefulness and acceptability. ${ }^{11}$

None of the six prospective studies included a control group. ${ }^{10-15}$ Five of these studies ${ }^{1012-15}$ were rated as poor quality for the following reasons: poor outcome measures, lack of blinding, no description of attrition and not using an interrupted time series design (multiple measurements before and after intervention).

One study measuring the acceptability of a well-being intervention ${ }^{11}$ was appraised as high quality due to clearly stating the aims and objectives, a clearly specified and appropriate research design for the aims and objectives of the research, a clear account of the process by which their findings were found, displaying enough data to support their interpretations and conclusions and the method of analysis being appropriate and adequately explained. ${ }^{9}$

None of the six prospective studies reported used theory in their approach to designing the intervention. ${ }^{10-15}$ One study ${ }^{12}$ mentioned theory in its interpretation of the data and related it to nurses adopting coping mechanisms as a response to $\operatorname{crises}^{24}$ (see table 1 ).

\section{Descriptive studies}

Details of the seven descriptive studies ${ }^{16-22}$ are provided in table 2. Six were related to the COVID-19 pandemic ${ }^{16-1820-22}$ and one was related to a nuclear disaster. ${ }^{19}$ All interventions targeted psychological well-being. Six interventions targeted the psychological well-being of HCWs, ${ }^{16-18}$ 20-22 and one studied disaster recovery support staff. ${ }^{19}$ The contents of these interventions included social support, ${ }^{16}$ provision of psychological support, ${ }^{17}{ }^{19}$ signposting to psychological support ${ }^{18} 2022$ and a mixture of physical and psychological support. ${ }^{21}$ None of these studies ${ }^{16-22}$ included outcome measures or provided any analysis. 


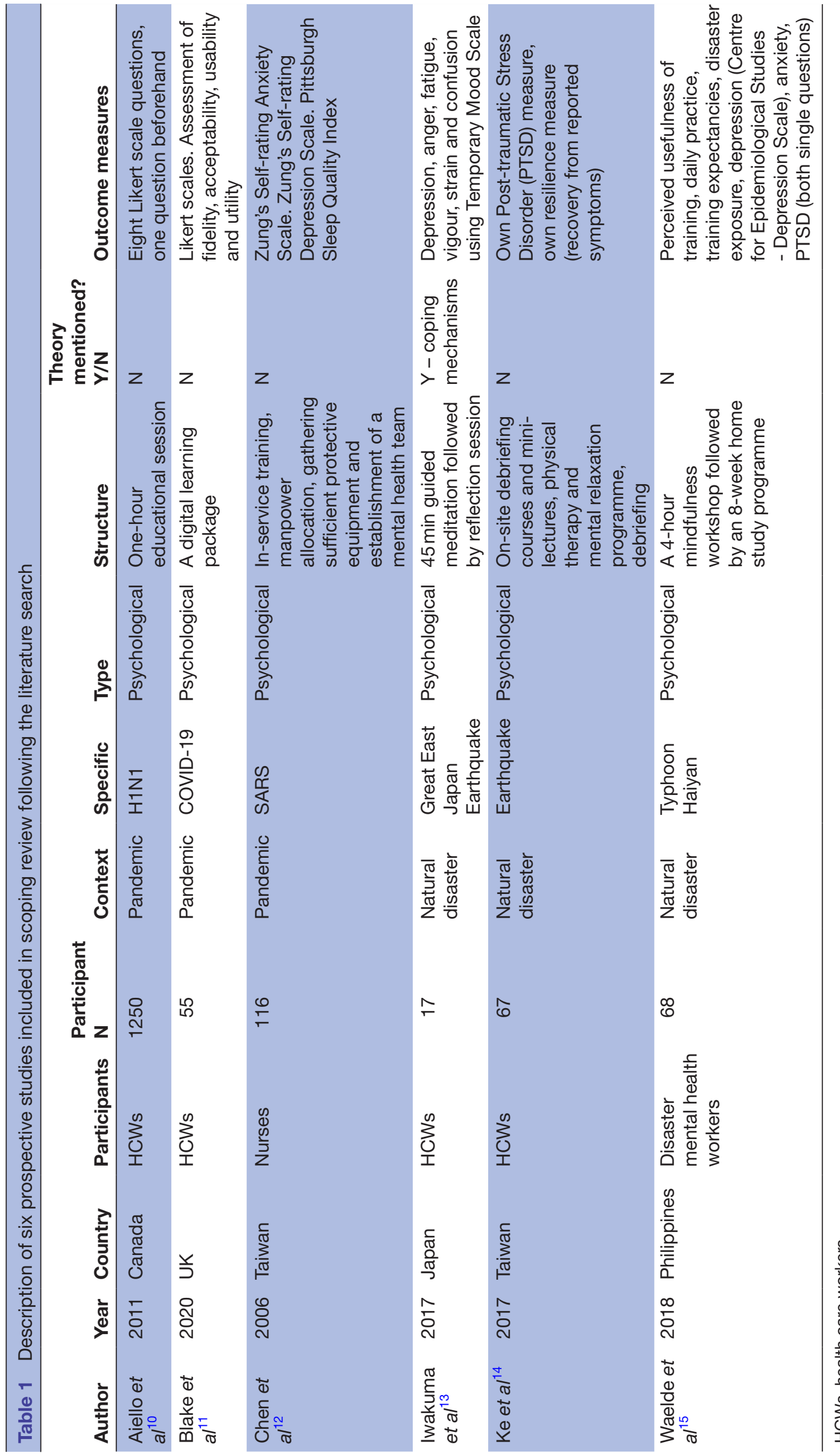

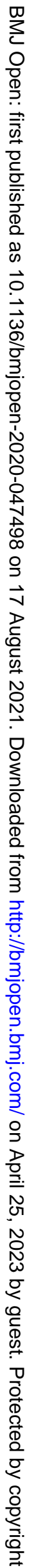




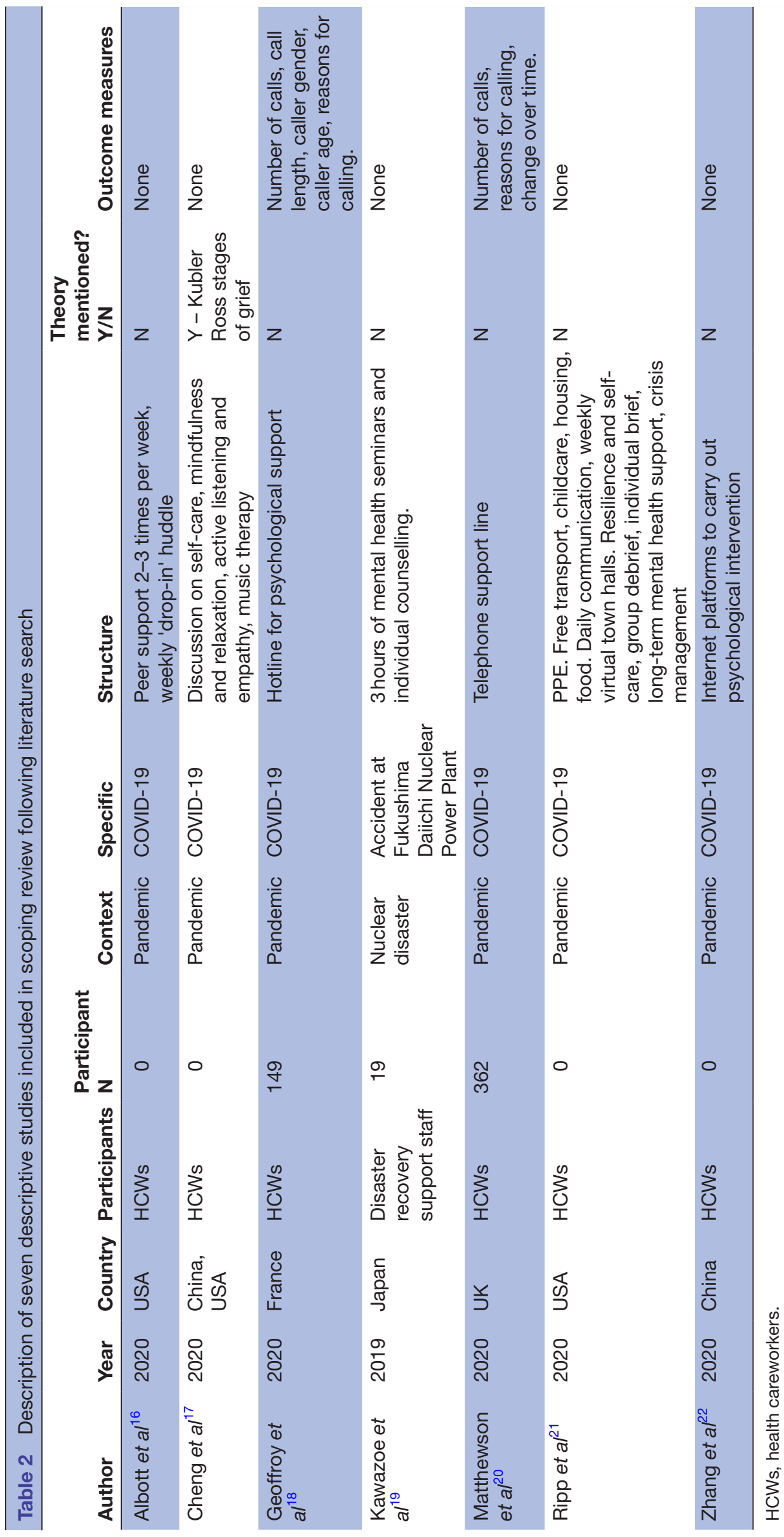

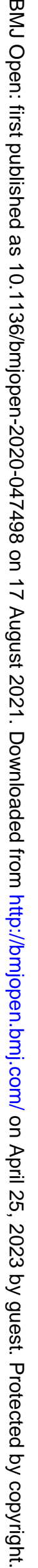


As these seven descriptive studies either did not include outcome measures ${ }^{1516182021}$ or statistical analysis, ${ }^{1719}$ no quality assessment tool was used.

None of the seven descriptive studies mentioned used theory in their approach to designing the intervention. ${ }^{16-22}$ One study ${ }^{16}$ mentioned theory in its discussion of their intervention, suggesting that HCWs may go through Kubler-Ross's ${ }^{25}$ seven stages of grief during and after the pandemic. No evidence is provided to support this claim (see table 2).

\section{DISCUSSION}

The primary finding from this scoping review is that no published evidence base exists regarding interventions for the well-being of doctors during pandemics and limited literature exists concerning the well-being of HCWs in general during crises. This limited evidence includes no RCTs, six prospective trials and seven descriptive studies published between 2006 and 2020. Of these prospective trials, all, with the exception of one, ${ }^{11}$ were deemed to be of poor quality. Blake $e t a l^{11}$ assessed fidelity, acceptability, usability and utility. Thus, it has not been proven to be effective in positively influencing the psychological well-being of HCWs. The seven descriptive studies did not include statistical analyses. Furthermore, although two studies mentioned theory to interpret their results, ${ }^{13} 17$ none of the included studies ${ }^{10-22}$ reported using theory to develop their interventions and therefore are not considered theory-based. ${ }^{26}$ Within the grey literature, although resources for the well-being of HCWs were available, the majority did not have peer-reviewed publications to support their use and were not designed specifically for use in a pandemic.

The strengths of this scoping review include utilisation of a transparent methodological approach supported by the application of an established methodological framework. ${ }^{7}$ Our use of a bibliographic manager (EndNote) meant all citations and articles were properly accounted for during the process. The literature search was comprehensive, and included seven academic bibliographic databases, three grey literature databases and one internet search engine. We adopted Medical Subject Headings in an effort to enhance the depth and accuracy of our searches. The data capture forms were pretested by all reviewers and revised prior to implementation. Finally, we attempted to contact relevant researchers via social media to uncover relevant literature. The limitations of this scoping review were that it only included articles published in English and as such, may have omitted well-being interventions developed for HCWs in other languages. Our search terms were designed to be exhaustive, but other search terms may exist that could produce further findings. Furthermore, although a grey literature search was undertaken there may be interventions used in practice or in local contexts that are effective but have not been published and so are unidentifiable.
The fact that no high-quality, theory-based interventions exist is problematic. Pandemics are crisis moments and time sensitive, demanding the majority of HCWs' time and attention. However, occurrences of infectious diseases are increasing, something that has been known for over a decade, ${ }^{27}$ and the negative effect of these outbreaks on HCW well-being has been known for still longer. ${ }^{3}{ }^{4}$ The importance of evidence for high-quality interventions that support HCW well-being cannot be overemphasised and represents a significant gap in the literature.

In light of the lack of available evidence, it is not possible to give recommendations regarding interventions for the well-being of HCWs during a pandemic or other crisis. However, recommendations can be given regarding the direction of future research into this topic. Well-being interventions are complex, and their development should be supported by the use of theory, as recommended by the General Medical Council. ${ }^{5}$ For example, incorporating the Behaviour Change Whee ${ }^{28}$ and Behaviour Change Taxonomy ${ }^{29}$ would characterise key mechanisms of an intervention which contribute to its success or failure. Examples of these could include adding objects to the environment such as well-being resources, providing social support such as an online group therapy session or the modelling of behaviours including instruction on how to perform a behaviour such as accessing support. ${ }^{29}$ Identifying mechanisms of action ensures that interventions are replicable and that active ingredients of these interventions are accurately pinpointed.

\section{CONCLUSION}

Thirteen interventions were found relating to interventions for the well-being of HCWs during a crisis. However, these were prospective and descriptive studies and were generally judged to be of poor quality during quality assessment. This scoping review highlights the need for high-quality, theory-based interventions for the well-being of HCWs during a pandemic.

Author affiliations

${ }^{1}$ School of Medicine, University of St Andrews, St Andrews, UK

${ }^{2}$ Centre for Medical Education, The University of Edinburgh, Edinburgh, UK

${ }^{3}$ General Practice and Primary Care, University of Glasgow, Glasgow, UK

${ }^{4}$ NHS Education for Scotland, Glasgow, UK

${ }^{5}$ Centre for Healthcare Education Research and Innovation, University of Aberdeen, Aberdeen, UK

${ }^{6}$ Centre for Medical Education, University of Dundee, Dundee, UK

${ }^{7}$ Monash Centre for the Scholarship in Health Education, Monash University, Clayton, Victoria, Australia

${ }^{8} \mathrm{NHS}$ Education for Scotland, Aberdeen, UK

${ }^{9} \mathrm{NHS}$ Education for Scotland, Edinburgh, UK

Twitter Patrick Cairns @kernzi, Lindsey Margaret Pope @LindseyMPope, Katie Gibson Smith @kgibsonsmith7, Lisi Gordon @lisigordon, Gillian Marion Scanlan @ gillianmscanlan, Tricia R Tooman @trtooman and Kim Walker @DrkimMeded

Acknowledgements The authors thank Vicki Cormie, librarian at University of St Andrews, for advice on conducting scoping reviews.

Contributors This study was part of a larger research project 'To develop evidence-based interventions to support doctors' wellbeing and promote resilience 
during COVID-19 (and beyond)', for which KW as principal investigators and PJ, LMP, GA, LG, KGS, AL, JEC and GMS as co-investigators conceived the work. PC, TRT and KBC were involved with the design of the work. PC prepared the original draft of the paper and GA, LMP, JF, GMS, TRT, LG, AL, JEC, KBC, KGS, PJ, JW and KW contributed to the refinement of the paper. All authors have read and approved the final paper and agree to be accountable for all aspects of the work. This study is guaranteed by the University of Aberdeen.

Funding This study was supported by Chief Scientist Office of Scotland, grant number COV/ABN/20/06.

Competing interests None declared.

Patient consent for publication Not required.

Ethics approval Ethical approval for this study was not required as it was a review and no participants were involved.

Provenance and peer review Not commissioned; externally peer reviewed.

Data availability statement Data sharing not applicable as no datasets generated and/or analysed for this study.

Supplemental material This content has been supplied by the author(s). It has not been vetted by BMJ Publishing Group Limited (BMJ) and may not have been peer-reviewed. Any opinions or recommendations discussed are solely those of the author(s) and are not endorsed by BMJ. BMJ disclaims all liability and responsibility arising from any reliance placed on the content. Where the content includes any translated material, BMJ does not warrant the accuracy and reliability of the translations (including but not limited to local regulations, clinical guidelines, terminology, drug names and drug dosages), and is not responsible for any error and/or omissions arising from translation and adaptation or otherwise.

Open access This is an open access article distributed in accordance with the Creative Commons Attribution Non Commercial (CC BY-NC 4.0) license, which permits others to distribute, remix, adapt, build upon this work non-commercially, and license their derivative works on different terms, provided the original work is properly cited, appropriate credit is given, any changes made indicated, and the use is non-commercial. See: http://creativecommons.org/licenses/by-nc/4.0/.

\section{ORCID iDs}

Patrick Cairns http://orcid.org/0000-0002-8480-8386

Lindsey Margaret Pope http://orcid.org/0000-0003-0899-9616

Joanne E Cecil http://orcid.org/0000-0003-4779-6037

Peter Johnston http://orcid.org/0000-0002-9446-8354

Anita Laidlaw http://orcid.org/0000-0003-1214-4100

Tricia R Tooman http://orcid.org/0000-0002-1227-2033

Kim Walker http://orcid.org/0000-0001-8873-2270

\section{REFERENCES}

1 Vindegaard N, Benros ME. COVID-19 pandemic and mental health consequences: systematic review of the current evidence. Brain Behav Immun 2020:89:531-42.

2 Luo M, Guo L, Yu M, et al. The psychological and mental impact of coronavirus disease 2019 (COVID-19) on medical staff and general public - A systematic review and meta-analysis. Psychiatry Res 2020;291:113190.

3 Chong M-Y, Wang W-C, Hsieh W-C, et al. Psychological impact of severe acute respiratory syndrome on health workers in a tertiary hospital. Br J Psychiatry 2004;185:127-33.

4 Maunder RG, Lancee WJ, Balderson KE, et al. Longterm psychological and occupational effects of providing Hospital healthcare during SARS outbreak. Emerg Infect Dis 2006;12:1924-32.

5 Craig P, Dieppe P, Macintyre S, et al. Developing and evaluating complex interventions: the new medical Research Council guidance. BMJ 2008;337:a1655.
6 Munn Z, Peters MDJ, Stern C, et al. Systematic review or scoping review? Guidance for authors when choosing between a systematic or scoping review approach. BMC Med Res Methodol 2018;18:143.

7 Arksey H, O'Malley L. Scoping studies: towards a methodological framework. Int J Soc Res Methodol 2005;8:19-32.

8 Tricco AC, Lillie E, Zarin W, et al. PRISMA extension for scoping reviews (PRISMA-ScR): checklist and explanation. Ann Intern Med 2018;169:467-73.

$9 \mathrm{NIH}$, National Heart, Lung and Blood Institute. Quality assessment tool for before-after (pre-post) studies with no control group, 2014. Available: https://www.nhlbi.nih.gov/health-topics/study-qualityassessment-tools

10 Aiello A, Khayeri MY-E, Raja S, et al. Resilience training for hospital workers in anticipation of an influenza pandemic. J Contin Educ Health Prof 2011;31:15-20.

11 Blake H, Bermingham F, Johnson G, et al. Mitigating the psychological impact of COVID-19 on healthcare workers: a digital learning package. Int J Environ Res Public Health 2020;17:2997.

12 Chen R, Chou K-R, Huang Y-J, et al. Effects of a SARS prevention programme in Taiwan on nursing staff's anxiety, depression and sleep quality: a longitudinal survey. Int J Nurs Stud 2006;43:215-25.

13 Iwakuma M, Oshita D, Yamamoto A, et al. Effects of breathing-based meditation on earthquake-affected health professionals. Holist Nurs Pract 2017;31:177-82.

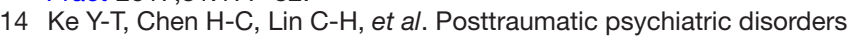
and resilience in healthcare providers following a disastrous earthquake: an interventional study in Taiwan. Biomed Res Int 2017;2017:1-7.

15 Waelde LC, Hechanova MRM, Ramos PAP, et al. Mindfulness and MANTRA training for disaster mental health workers in the Philippines. Mindfulness 2018;9:1181-90.

16 Albott CS, Wozniak JR, McGlinch BP, et al. Battle buddies: rapid deployment of a psychological resilience intervention for health care workers during the COVID-19 pandemic. Anesth Analg 2020;131:43-54.

17 Cheng P, Xia G, Pang P, et al. COVID-19 epidemic peer support and crisis intervention via social media. Community Ment Health $J$ 2020;56:786-92.

18 Geoffroy PA, Le Goanvic L, V, Sabbagh O, et al. Psychological support system for hospital workers during the Covid-19 outbreak: rapid design and implementation of the Covid-Psy Hotline. Front Psychiatry 2020;11:511.

19 Kawazoe I, Kidachi R, Norikane K, et al. Stress management for disaster recovery support staff assisting nuclear disaster evacuees. Radiat Prot Dosimetry 2019;184:547-9.

20 Matthewson J, Tiplady A, Gerakios F, et al. Implementation and analysis of a telephone support service during COVID-19. Occup Med 2020;70:375-81.

21 Ripp J, Peccoralo L, Charney D. Attending to the emotional wellbeing of the health care workforce in a New York City health system during the COVID-19 pandemic. Acad Med 2020;95:1136-9.

22 Zhang J, Wu W, Zhao X, et al. Recommended psychological crisis intervention response to the 2019 novel coronavirus pneumonia outbreak in China: a model of West China Hospital. Precis Clin Med 2020;3:3-8.

23 Evans D. Hierarchy of evidence: a framework for ranking evidence evaluating healthcare interventions. J Clin Nurs 2003;12:77-84.

24 Aguilera DC. Crisis intervention: theory and methodology. 6th edn. St. Louis: Mosby, 1994.

25 Kubler-Ross E. On death and dying. New York: Macmillan Publishing, 1969.

26 Michie S, Prestwich A. Are interventions theory-based? Development of a theory coding scheme. Health Psychol 2010;29:1-8.

27 Jones KE, Patel NG, Levy MA, et al. Global trends in emerging infectious diseases. Nature 2008;451:990-3.

28 Michie S, Richardson M, Johnston M, et al. The behavior change technique taxonomy (V1) of 93 hierarchically clustered techniques: building an international consensus for the reporting of behavior change interventions. Ann Behav Med 2013:46:81-95.

29 Michie S, van Stralen MM, West R. The behaviour change wheel: a new method for characterising and designing behaviour change interventions. Implement Sci 2011;6:42. 